\title{
Hérnias incisionais no pós-operatório de correção de aneurisma de aorta abdominal
}

\author{
Postoperative incisional hernias after open abdominal aortic aneurysm repair
}

Fábio Hüsemann Menezes¹, Ana Terezinha Guillaumon²

\begin{abstract}
Resumo
Contexto: A incidência de hérnia incisional no pós-operatório da correção aberta de aneurisma de aorta abdominal é alta, variando de 10 a 37\% e mais de três vezes mais comum do que em pacientes submetidos à correção para doença obstrutiva aorto-ilíaca.

Objetivo: Apresentar a incidência de hérnia incisional em um grupo de pacientes acompanhados no pós-operatório da correção aberta de aneurisma de aorta abdominal.

Métodos: Série de casos em uma população de 144 pacientes operados por aneurisma de aorta abdominal, entre junho de 1989 e junho de 2010 , e que estão em acompanhamento regular no Ambulatório de Moléstias Vasculares.

Resultados: O seguimento médio dos pacientes foi de 63 meses (1 a 238). A idade média foi de 67 anos (45 a 91) e o tamanho médio dos aneurismas foi de 6,54 cm. Foram realizadas 130 laparotomias medianas xifo-púbicas e 13 acessos extraperitoniais pelo flanco esquerdo. Nestes pacientes, a incidência de hérnia incisional foi de 18,5 e 7,7\%, respectivamente, para incisões na linha média ou no flanco ( $p=0,315)$. Um paciente apresentou abaulamento da musculatura oblíqua por denervação. Foi realizada uma laparotomia transversa, que não apresentou hérnia no pós-operatório tardio.

Conclusões: A incidência de hérnia incisional na cirurgia aberta para correção de aneurisma de aorta abdominal é alta, ocorre com maior frequência em incisões da linha média e tem relação direta com a técnica empregada para o fechamento da aponeurose, exigindo do cirurgião atenção especial para este tempo cirúrgico para evitar a causa mais comum de reoperação em tal grupo de pacientes.
\end{abstract}

Palavras-chave: aneurisma da aorta; /cirurgia; hérnia ventral.

\begin{abstract}
Background: The incidence of incisional hernia in the post operatory of patients submitted to open abdominal aortic aneurysm repair is high, ranging from 10 to 37\%, and is more than three times higher than the incidence of hernias in patients operated for aorto-iliac occlusion.

Objective: To evaluate the incidence of incisional hernia in patients followed-up after open repair of an abdominal aortic aneurysm.

Methods: Case series of 144 patients submitted to open abdominal aortic aneurysm repair between June, 1989 and June, 2010 and routinely followed-up at the vascular outpatient clinic.

Results: The mean follow-up was 63 months (1 to 238). The mean age was 67 years-old (from 45 to 91). The mean aneurysm diameter was $6.54 \mathrm{~cm}$. There were 130 mid line laparotomies and 13 extraperitonial left flank incisions. The incidence of incisional hernia was respectively 18.5 and $7.7 \%$ $(p=0.315)$. One patient presented bulging of the flank musculature. There was one transverse incision, which did not present hernia during the followup period.

Conclusions: The incidence of incisional hernia is high in patients submitted to open abdominal aortic aneurysm repair, it is more common in midline incisions and is related to the closing surgical technique. The surgeon must be attentive to this surgical step in order to avoid the most common cause of reoperation for this group of patients.
\end{abstract}

Keywords: aortic aneurysm; /surgery; hernia, ventral.

Trabalho realizado no Hospital de Clínicas, Disciplina de Moléstias Vasculares da Faculdade de Ciências Médicas da Universidade Estadual de Campinas (Unicamp) - Campinas (SP), Brasil.

${ }^{1}$ Doutor; Professor-Assistente da Disciplina de Moléstias Vasculares do Departamento de Cirurgia da Faculdade de Ciências Médicas da Unicamp - Campinas (SP), Brasil.

${ }^{2}$ Livre-Docente; Professora-Associada da Disciplina de Moléstias Vasculares do Departamento de Cirurgia da Faculdade de Ciências Médicas da Unicamp - Campinas (SP), Brasil.

Fonte de financiamento: nenhuma.

Conflito de interesses: nada a declarar.

Submetido em: 05.09.11. Aceito em: 16.01.12.

J Vasc Bras. 2012;11(3):182-186. 


\section{Introdução}

A alta incidência de hérnia incisional após a correção cirúrgica do aneurisma de aorta abdominal é bem conhecida e varia de 10 a $37 \%$. Quando comparadas com a doença arterial obstrutiva aorto-ilíaca, tanto a incidência de hérnia incisional, assim como a de hérnia inguinal, são descritas como três a nove vezes maiores em pacientes com doença aneurismática ${ }^{1-6}$. Esta tendência está relacionada a alterações da matriz extracelular do tecido conjuntivo dos portadores de aneurismas.

Há evidências na literatura de que os pacientes portadores de aneurisma de aorta apresentam menor quantidade de elastina na camada média da parede arterial e, embora haja maior deposição de colágeno na camada adventicial, este perde sua estrutura normal em forma de rede, depositandose de forma desordenada e densa, à semelhança de tecido fibrótico. Essas alterações levam à perda das características elásticas da parede, o que facilita a distensão do tecido.

O mecanismo por trás destas alterações está associado ao desequilíbrio do metabolismo da matriz extracelular, com maior atividade proteolítica, evidenciada pela elevação de atividade das metaloproteinases e diminuição dos inibidores teciduais das mesmas. Por um lado, a maior atividade inflamatória observada na parede arterial pode estar associada ao acúmulo de fragmentos proteicos e, por outro, pode agravar o estado de desequilíbrio. Esta atividade é agravada pelos fatores de risco, notadamente o tabagismo. Também há suficiente evidência de que há maior incidência de aneurismas e hérnias nos familiares dos pacientes afetados, sugerindo uma predisposição genética, embora não tenha sido identificado nenhum gene específico responsável pela doença, com exceção dos relacionados à Síndrome de Marfan (defeito na fibrilina) e Ehlers-Danlos (defeito no colágeno tipo III). O desequilíbrio no metabolismo extracelular pode ser o responsável pelo desbalanço na relação dos colágenos I e III, encontrada nos pacientes portadores de hérnias ${ }^{7-10}$.

\section{Objetivo}

Apresentar a incidência de hérnia incisional em um grupo de pacientes acompanhados no pós-operatório da correção aberta de aneurisma da aorta abdominal.

\section{Casuística e métodos}

Estudo do tipo série de casos, em uma população de 144 pacientes operados por aneurisma de aorta abdominal, entre junho de 1989 e junho de 2010, e que estão em acompanhamento regular no Ambulatório de Moléstias Vasculares do Hospital de Clínicas da Universidade Estadual de Campinas (Unicamp). Desde que o sistema do centro cirúrgico foi informatizado, em 1995, e até dezembro de 2010, foram operados eletivamente 403 pacientes, mas a maioria perdeu o seguimento regular. Os dados referentes ao procedimento cirúrgico foram extraídos dos prontuários médicos de forma retrospectiva até 2006 e prospectiva a partir de 2007, quando foi criado um ambulatório específico para o seguimento de tais pacientes. Os dados referentes à presença de hérnia incisional foram coletados prospectivamente, por meio de interrogatório complementar e exame físico dos pacientes durante as consultas de retorno. Os dados foram tabulados em programa de planilha eletrônica (Excel, Microsoft 2003, Redmond, WA) e submetidos ao estudo estatístico (SPSS v.15, Chicago, Il). O estudo incluiu uma análise descritiva dos dados e os testes do $\chi^{2}$ e exato de Fischer para comparação dos prováveis fatores de risco associados à presença de hérnia incisional. Um valor de $\mathrm{p}<0,05$ foi considerado significativo. O protocolo de pesquisa foi aprovado pelo Comitê de Ética em Pesquisa da Instituição, recebendo aprovação sob número 256/2011.

\section{Resultados}

O seguimento médio dos pacientes foi de 63 meses ( 1 a 238). A idade média foi de 67 anos ( 45 a 91) e o tamanho médio dos aneurismas foi de $6,54 \mathrm{~cm}(3,5 \mathrm{a} 11)$. O aneurisma com $3,5 \mathrm{~cm}$ foi operado devido à dilatação da ilíaca comum de $5,5 \mathrm{~cm}$. Os fatores de risco para aterosclerose deste grupo estão descritos na Tabela 1.

Foram realizadas 130 laparotomias medianas xifopúbicas e 13 acessos extraperitoniais pelo flanco esquerdo. Nesses pacientes, a incidência de hérnia incisional foi de 18,5 e 7,7\%, respectivamente, para incisões na linha média ou no flanco $(\mathrm{p}=0,315)$. Um paciente apresentou abaulamento da musculatura oblíqua por denervação. Foi realizada uma incisão transversa em uma paciente apresentando obesidade mórbida, com índice de massa corpórea (IMC) de $41 \mathrm{~kg} / \mathrm{m}^{2}$.

No pós-operatório precoce (menor do que sete dias) houve três eviscerações, todas em laparotomias medianas e em pacientes não-obesos e sem alterações pulmonares. Um destes pacientes com antecedente de etilismo crônico entrou em síndrome de abstinência no pós-operatório. Os três evoluíram sem hérnia no seguimento tardio após a ressutura da parede (seguimento de seis e dez meses, seis anos). 
Houve cinco deiscências de subcutâneo sem desenvolvimento de hérnias incisionais no seguimento, incluindo a paciente submetida à incisão transversa, devido à obesidade.

O fechamento da laparotomia foi realizado com sutura contínua, utilizando fio absorvível de polidiaxonona (PDS II ${ }^{\mathrm{TM}}$, Ethicon, West Somerville, NJ) de espessura 1 (18 doentes), ou com sutura contínua, utilizando fio de polipropileno (Prolene ${ }^{\mathrm{TM}}$, Ethicon, West Somerville, NJ) espessura zero, interrompida a cada $8 \mathrm{~cm}$ (38 doentes). Em cinco pacientes, o fechamento foi realizado com sutura contínua com fio de poliglactina 910 (Vicryl $^{\mathrm{TM}}$, Ethicon, West Somerville, NJ) de espessura 1. Em todos os casos foram utilizados pontos separados subtotais internos (não interessando o peritônio parietal e a pele) a cada 5 a $8 \mathrm{~cm}$, com fio de poliglactina de espessura 2 . As incisões extraperitoniais foram fechadas com sutura contínua com fio de poliglactina de espessura zero. Em 72 (50,7\%) pacientes, não constava na descrição de cirurgia o tipo de fio utilizado para o fechamento da incisão abdominal. A incidência de hérnia incisional nos pacientes com fechamento com fio de polipropileno foi de $8,3 \%$ e com fio de polidiaxonona foi $28,6 \%$, uma diferença grande, embora não tenha alcançado significância estatística $(\mathrm{p}=0,083)$.

$\mathrm{O}$ antecedente de herniorrafia inguinal/umbilical foi de $12,6 \%$, sendo que a presença de hérnia incisional, no grupo que já havia sido operado para correção de outra hérnia, foi de $33 \%$ e, no grupo que não havia apresentado hérnia em outra localização, $15,2 \%(\mathrm{p}=0,058)$, também uma diferença grande embora não tenha alcançado significância estatística.

O IMC acima ou abaixo de $30 \mathrm{~kg} / \mathrm{m}^{2}$ também não alterou a incidência de hérnia incisional (40 vs. 19,6\%, $\mathrm{p}=0,272$ ), embora haja tendência a uma maior incidência de hérnia incisional no grupo com IMC acima de $30 \mathrm{~kg} / \mathrm{m}^{2}$, mas que, na presente casuística, constituiu um grupo muito pequeno de pacientes $(\mathrm{n}=10)$.

O tamanho do aneurisma maior ou menor do que $6,5 \mathrm{~cm}$ de diâmetro não influenciou a incidência de hérnia incisional (18,3 vs. 23,5\%, $\mathrm{p}=0,65)$.

Não houve diferença quando se comparou a presença de hérnia incisional com o tempo cirúrgico acima ou abaixo de quatro horas $(17,2$ vs. $25,4 \%, \mathrm{p}=0,501)$.

O volume de sangramento acima ou abaixo de $1.000 \mathrm{~mL}$ também não influenciou a incidência de hérnia incisional $(16,4$ vs. $27,3 \%, \mathrm{p}=0,278)$.

A ausência ou a presença de pneumopatia sintomática também não influenciou a presença de hérnia incisional $(19,3$ vs. $24,1 \%, \mathrm{p}=0,62)$.
Tabela 1. Dados demográficos e fatores de risco para aterosclerose da população estudada $(n=144)$.

\begin{tabular}{|c|c|}
\hline Parâmetro & $\mathrm{n}(\%)$ \\
\hline \multicolumn{2}{|l|}{ Sexo } \\
\hline Masculino & $121(84,0)$ \\
\hline Feminino & $23(16,0)$ \\
\hline Idade em anos (mínima-máxima) & $66,8(45-91)$ \\
\hline \multicolumn{2}{|l|}{ Raça } \\
\hline Branca & $135(93,0)$ \\
\hline Negra & $8(5,6)$ \\
\hline Amarela & $1(1,4)$ \\
\hline \multicolumn{2}{|l|}{ Cardiopatia } \\
\hline Assintomático, ECG normal & $94(65,3)$ \\
\hline Assintomático, IAM antigo & $15(10,4)$ \\
\hline Sintomático e controlado (angina, arritmia, ICC) & $32(22,2)$ \\
\hline Desconhecido & $3(2,1)$ \\
\hline \multicolumn{2}{|l|}{ Pneumopatia } \\
\hline Assintomático & $105(72,9)$ \\
\hline Sintomático (PFP 65 a 80\%) & $30(20,8)$ \\
\hline Sintomático (PFP 35 a 65\%) & $5(3,5)$ \\
\hline Dependente $\mathrm{O}_{2}, \mathrm{PFP}<35 \%$ & $1(0,7)$ \\
\hline Desconhecido & $3(2,1)$ \\
\hline \multicolumn{2}{|l|}{ Nefropatia } \\
\hline Sem doença, $\mathrm{Cr}<1,5$ & $122(84,7)$ \\
\hline $\operatorname{Cr} 1,5<3$ & $17(11,8)$ \\
\hline $\mathrm{Cr} 3<6$ & $1(0,7)$ \\
\hline Desconhecido & $4(2,8)$ \\
\hline \multicolumn{2}{|l|}{ Doença cerebrovascular } \\
\hline Assintomático sem sopros & $124(86,1)$ \\
\hline Assintomático com estenose diagnosticada & $4(2,8)$ \\
\hline AIT & $1(0,7)$ \\
\hline AVC & $10(6,9)$ \\
\hline Desconhecido & $5(3,5)$ \\
\hline \multicolumn{2}{|l|}{ Hipertensão arterial sistêmica } \\
\hline Sem & $42(29,2)$ \\
\hline Controlada uma droga & $49(34,0)$ \\
\hline Controlada duas drogas & $41(28,5)$ \\
\hline Não controlada ou uso $>2$ drogas & $9(6,3)$ \\
\hline Desconhecido & $3(2,0)$ \\
\hline \multicolumn{2}{|l|}{ Diabetes melito } \\
\hline Sem & $123(85,4)$ \\
\hline Uso hipoglicemiante oral & $15(10,4)$ \\
\hline Uso de insulina & $3(2,1)$ \\
\hline Desconhecido & $3(2,1)$ \\
\hline \multicolumn{2}{|l|}{ Tabagismo } \\
\hline Nunca fumou & $14(9,7)$ \\
\hline Parou de fumar >10 anos & $26(18,1)$ \\
\hline Parou de fumar $<10$ anos e $>1$ ano & $27(18,8)$ \\
\hline Parou de fumar $<1$ ano & $10(6,9)$ \\
\hline Fumante ativo & $65(45,1)$ \\
\hline Desconhecido & $2(1,4)$ \\
\hline
\end{tabular}


A presença de tabagismo nos últimos dez anos ou a abstenção deste por mais de dez anos também não alterou a incidência de hérnia incisional ( $18 v s .30 \%, \mathrm{p}=0,29$ ).

\section{Discussão}

A incidência de hérnia incisional em pacientes submetidos à correção aberta para aneurismas de aorta abdominal é alta, variando entre 10 e $37 \%^{1-6}$. Na presente casuística, encontrou-se uma incidência $(18,5 \%)$ concordante com a literatura. A literatura revela que uma pequena parcela de pacientes se queixa da presença de hérnia incisional e, entre aqueles diagnosticados, poucos desejam ser submetidos à correção cirúrgica ${ }^{3,11,12}$. No entanto, a hérnia incisional constitui a principal indicação para reoperação após a correção aberta de um aneurisma de aorta abdominal, sendo muito mais comum do que as complicações relacionadas à prótese vascular ${ }^{13}$. O diagnóstico da hérnia incisional, quando realizado pelo exame físico, é menos frequente do que o auxiliado por exames de imagem, notadamente a ultrassonografia da parede abdominal ou a tomografia computadorizada do abdômen ${ }^{11,14}$.

O motivo da alta incidência de hérnias incisionais nestes pacientes é atribuído a um provável defeito no colágeno tecidual ${ }^{7}$, uma vez que diversos autores observaram que a incidência de hérnia incisional em operados para a correção de aneurismas de aorta é aproximadamente três vezes maior do que nos pacientes operados para doença aorto-ilíaca oclusiva ${ }^{1-6}$, assim como a incidência de aneurismas é aproximadamente três vezes maior em parentes de primeiro grau de pacientes operados, quando comparada com a do Grupo Controle, sugerindo um defeito comum $^{15,16}$. Há grande interesse na literatura em encontrar-se um defeito genético que poderia justificar o aparecimento do aneurisma de aorta, assim como de hérnias de parede abdominal. No entanto, não se conseguiu correlacionar diretamente um polimorfismo genético a um defeito evidente na estrutura do colágeno ou da elastina. Desta forma, acredita-se que estas doenças degenerativas são resultado da associação de múltiplos fatores, tanto genéticos como externos, levando ao processo inflamatório e à degradação da estrutura da parede vascular e abdominal $^{7-10}$. Observa-se, na presente casuística, que menos de $10 \%$ dos pacientes nunca foram fumantes, sendo que $70,8 \%$ deles eram fumantes ativos ou haviam parado há menos de dez anos, reforçando o achado da literatura de que o tabagismo é um importante agente externo associado ao aneurisma de aorta abdominal e aos defeitos de parede abdominal ${ }^{8}$. No entanto, na presente casuística, não se encontrou diferença estatística na incidência de hérnia incisional entre os fumantes e não-fumantes.

A diferença encontrada nos resultados, sugerindo maior incidência de hérnia incisional em indivíduos com a presença de hérnias em outras localizações, assim como o tipo de fio utilizado, provavelmente não alcançou significado estatístico em consequência do baixo número de indivíduos envolvidos nas análises. Na presente casuística, sugere-se maior incidência de hérnia incisional nos pacientes que também apresentaram hérnias em outras localizações (inguinal e umbilical). A literatura relata a alta incidência de hérnia inguinal em pacientes operados por aneurisma de aorta, mas não discute se a incidência de hérnias incisionais foi maior no grupo que também apresentava outras hérnias $^{4-6}$. Este achado poderia auxiliar a detectar um grupo com defeito do colágeno estrutural e auxiliar a direcionar estudos genéticos de polimorfismos.

Há discordância na literatura quanto à influência do tamanho do aneurisma de aorta (maior ou igual a $6,5 \mathrm{~cm}$ ) e à incidência de hérnia incisional ${ }^{4,5}$, na presente casuística não se encontrou associação entre o tamanho do aneurisma e a incidência de hérnia incisional, assim como não se encontrou associação com o tempo e o sangramento operatórios e com a presença de pneumopatia sintomática, fatores que poderiam influenciar favorecendo o aparecimento de uma hérnia incisional.

A literatura sugere que a técnica de fechamento influencia a incidência de hérnia incisional. Israelsson ${ }^{17}$ demonstrou que, se os pontos não forem espaçados corretamente na aponeurose, assim como não abrangerem largura suficiente de tecido, há maior incidência de hérnia incisional. Para esta avaliação, foi feita a relação entre o comprimento da incisão e o de fio de sutura utilizado para o fechamento. Concluiu-se que uma relação menor do que quatro vezes resultaria em pontos muito espaçados ou com pouca abrangência do tecido, favorecendo o aparecimento de hérnia incisional. $\mathrm{O}$ uso de incisões transversas ${ }^{18} \mathrm{e}$ lombares $^{14}$ diminui a incidência de hérnia incisional, mas não a evita completamente. Mesmo o uso rotineiro da tela de polipropileno no fechamento da incisão mediana não evita por completo o aparecimento de hérnia incisional ${ }^{12,19,20}$. A videolaparoscopia utilizada em pacientes com IMC acima de $32 \mathrm{~kg} / \mathrm{m}^{2}$ apresentou bom resultado, com ausência de hérnias incisionais pelos orifícios dos portais ${ }^{21}$. Na presente casuística, em apenas metade dos casos, foi descrito o fio de fechamento, falha encontrada de forma consistente ao longo dos anos. Também observou-se maior uso do fio de polidiaxonona entre 2006 e 2007, contrastando com o maior uso do fio de polipropileno em 2009 e 2010. Nestes anos, 
a falta de relato do fio utilizado foi menor (20\%). Levando em consideração as limitações mencionadas, sugere-se que haja diferença entre o fechamento da aponeurose com fio de polipropileno e de polidiaxonona, mas atribui-se a maior incidência de hérnia incisional com o fio absorvível à técnica de fechamento e não ao material do fio em si, pois o fio de polidiaxonona possui uma agulha maior, induzindo o cirurgião a aumentar o espaçamento entre os pontos, o que está de acordo com o trabalho de Israelsson ${ }^{17}$.

\section{Conclusão}

A incidência de hérnia incisional na cirurgia aberta para correção de aneurisma de aorta abdominal é alta, ocorre com maior frequência em incisões da linha média e tem relação direta com a técnica empregada para o fechamento da aponeurose, exigindo do cirurgião atenção especial para este tempo cirúrgico para evitar a causa mais comum de reoperação neste grupo de pacientes.

\section{Referências}

1. Takagi $H$, Sugimoto $M$, Kato $T$, Matsuno $Y$, Umemoto $T$. Postoperative Incision Hernia in Patients with Abdominal Aortic Aneurysm and Aortoiliac Occlusive Disease: A Systematic Review. Eur J Vasc Endovasc Surg. 2007;33:177-81. http://dx.doi. org/10.1016/j.ejvs.2006.07.009

2. Stevick CA, Long JB, Jamasbi B, Nash M. Ventral hernia following abdominal aortic reconstruction. Am J Surg. 1988;54:287-9. PMid:3364866.

3. Holland AJA, Castleden WM, Norman PE, Stacey MC. Incisional Hernias are More Common in Aneurysmal Arterial Disease. Eur J Vasc Endovasc Surg. 1996;12:196-200. http://dx.doi.org/10.1016/ S1078-5884(96)80106-7

4. Adye B, Luna G. Incidence of abdominal wall hernia in aortic surgery. Am J Surg. 1998;175:400-2. PMid:9600287.

5. Papadimitriou D, Pitoulias G, Papaziogas B, et al. Incidence of abdominal wall hernias in patients undergoing aortic surgery for aneurysm or occlusive disease. Vasa. 2002;31:111-4. PMid:12099141.

6. Raffetto JD, Cheung Y, Fisher JB, et al. Incision and abdominal wall hernias in patients with aneurysm or occlusive aortic disease. J Vasc Surg. 2003;37:1150-4. http://dx.doi.org/10.1016/ S0741-5214(03)00147-2

7. Sandford RM, Bown MJ, London NJ, Sayers RD. The genetic basis of abdominal aortic aneurysms: a review. Eur J Vasc Endovasc Surg. 2007;33:381-90. http://dx.doi.org/10.1016/j.ejvs.2006.10.025

8. Antoniou GA, Georgiadis GS, Antoniou SA, Granderath FA, Giannoukas AD, Lazarides MK. Abdominal aortic aneurysm and abdominal wall hernia as manifestations of a connective tissue disorder. J Vasc Surg. 2011;54:1175-81. http://dx.doi.org/10.1016/j. jvs.2011.02.065

9. Lindemana JHN, Ashcroftb BA, Beenakkerb JWM, et al. Distinct defects in collagen microarchitecture underlie vessel-wall failure in advanced abdominal aneurysms and aneurysms in Marfan syndrome. PNAS. 2010;107:862-5. PMid:20080766.
10. Urbonaviciusa S, Lindholta IS, Delboscc S, et al. Proteins associated with the size and expansion rate of the abdominal aortic aneurysm wall as identified by proteomic analysis. Interact Cardiovasc Thorac Surg. 2010;11:433-41. PMid:20675398. http://dx.doi. org/10.1510/icvts.2010.238139

11. Rodriguez HE, Matsumura JS, Morasch MD, Greenberg RK, Pearce WH. Abdominal Wall Hernias After Open Abdominal Aortic Aneurysm Repair: Prospective Radiographic Detection and Clinical Implications. Vasc Endovascular Surg. 2004;38:237-40. PMid:15181505.

12. Bevis PM, Windhaber RAJ, Lear PA, Poskitt KR, Earnshaw J), Mitchell DC. Randomized clinical trial of mesh vs. sutured wound closure after open abdominal aortic aneurysm surgery. BJS. 2010;97:1497-502. PMid:20603858.

13. De Bruin JL, Baas AF, Jaap B, et al. Long-Term Outcome of Open or Endovascular Repair of Abdominal Aortic Aneurysm. N Eng J Med. 2010;362:1881-9. PMid:20484396.

14. Salameh JR, Salloum EJ. Lumbar Incisional Hernias: Diagnostic and Management Dilemma. Journal of the Society of Laparoscopic Surgeons. 2004;8:391-4. PMid:15554289.

15. Barbosa RD, Denardi MR, Rossini Sobrinho JR, Zorn WGW, Van Bellen B. Rastreamento com ultra-sonografia abdominal em irmãos de pacientes com aneurisma de aorta abdominal. Cir Vasc Angiol. 1995;11:68-71. PMid:16275443.

16. Ogata T, MacKean GL, Cole W, et al. The lifetime prevalence of abdominal aortic aneurysms among siblings of aneurysm patients is eightfold higher than among siblings of spouses: An analysis of 187 aneurysm families in Nova Scotia Canada. J Vasc Surg. 2005;42:8917. http://dx.doi.org/10.1016/j.jvs.2005.08.002

17. Israelsson LA. Incisional Hernias in Patients with Aortic Aneurysmal Disease: the Importance of Suture Technique. Eur I Vasc Endovas Surg. 1999;17:133-5. http://dx.doi.org/10.1053/ejvs.1998.0726

18. Fassiadis $N$, Roidl $M$, Hennig $M$, South $L M$, Andrews $S M$. Randomized clinical trial of vertical or transverse laparotomy for abdominal aortic aneurysm repair. BJS. 2005;92:508. PMid:16175532.

19. Rogers M, McCarthy R, Earnshaw Jj. Prevention of Incisional Hernia after Aortic Aneurysm Repair. Eur J Vasc Endovasc Surg. 2003;26:519-22. http://dx.doi.org/10.1016/S1078-5884(03)00383-6

20. O'Hare JL, Ward J, Earnshaw JJ. Late Results of Mesh Wound Closure after Elective Open Aortic Aneurysm Repair. Eur J Vasc Endovasc Surg. 2007;33:412-3. http://dx.doi.org/10.1016/j.ejvs.2006.11.015

21. Coscas R, Coggia M, Di Centa I, Javerliat I, Cochennec F, Brissonniere OG. Laparoscopic Aortic Surgery in Obese Patients. Ann Vasc Surg. 2009;23:717-21. http://dx.doi.org/10.1016/j.avsg.2009.01.003

Correspondência Fábio Hüsemann Menezes Rua Deusdeti Martins Gomes, 122 CEP 13084-723 - Campinas (SP), Brasil E-mail:fmenezes@mpc.com.br

Contribuições dos autores Concepção e desenho do estudo: FHM Análise e interpretação dos dados: FHM Coleta de dados: FHM Redação do artigo: FHM Revisão crítica do texto: FHM, ATG Aprovação final do artigo*: FHM, ATG Análise estatística: N/A Responsabilidade geral pelo estudo: FHM, ATG *Todos os autores leram e aprovaram a versão final submetida ao I Vasc Bras. 